\title{
Statistical Distribution of Coulomb Blockade Peak Heights in Adiabatically Pumped Quantum Dots
}

\author{
M. Blaauboer and E.J. Heller \\ Department of Physics, Harvard University, Cambridge, Massachusetts 02138
}

(Dated: October 31, 2018)

\begin{abstract}
We study adiabatic quantum pumping in the resonant tunneling regime of a nearly-closed quantum dot, which is coupled to two leads via tunneling barriers. Using small cyclic variations of the tunneling rates of the barriers as the pumping mechanism, a current is obtained which depends sensitively on the system parameters and exhibits peaks due to Coulomb blockade. The distribution of the peak heights is found for temperatures $\Gamma \ll k_{B} T \ll \Delta$, with $\Gamma$ the total decay width into the leads and $\Delta$ the single-particle level spacing of the dot, and their average height is predicted to increase by a factor of $\frac{5 \sqrt{2}}{18} \pi \approx 1.2$ upon breaking time-reversal symmetry. This is the pumping analog of the statistical theory of Coulomb blockade conductance peaks.
\end{abstract}

In recent years, the phenomenon of adiabatic quantum pumping in quantum dots has attracted a lot of theoretical and experimental attention. It is the generation of a d.c. current in the absence of a bias voltage by periodic modulations of two or more parameters, such as the shape of the dot or a magnetic field, that modify the quantum-mechanical properties of the system. The idea of adiabatic pumping was pioneered by Thouless [1] for electrons at zero temperature in an isolated one-dimensional periodic potential. Using cyclic variations of the potential, electrons can be pumped one by one through the system. Following early realizations of electron pumps based on "classical" sequential pumping mechanisms [2, 3], attention is now focussed on adi-

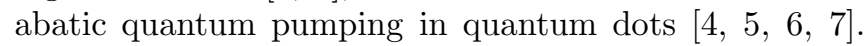
A quantum dot is a small metallic or semiconducting island, confined by gates and connected to electron reservoirs (leads) through quantum point contacts [8]. Almost all investigations of quantum pumping to date, including the first experimental observation [6], involved open quantum dots. Only very recently, quantum pumping in closed dots has for the first time been considered. In Ref. [7], Levinson et al. study adiabatic pumping mediated by resonant transmission through a quantum dot which is isolated from the leads through tunneling point contacts. They predict a quantized pumped current at zero temperature, if the two tunneling barriers are varied in such a way that the loop which describes the pumping cycle in parameter space encircles the entire resonance line.

Here we also consider adiabatic quantum pumping via resonant transmission through a nearly-closed quantum dot at low temperatures, but in a new regime, employing small periodic changes in the dot potential. This pumping mechanism differs from Ref. [7] in two significant ways: firstly, the tunneling rates are only slightly perturbed around a point of the resonance line, and as a result the pumped current is not quantized. Secondly, for such small modulations of the potential (linear response), which correspond to small perturbations of the energy level spectrum of the dot, the pumped current ex- hibits Coulomb blockade oscillations as a function of the Fermi energy of the dot. These are of the same origin as the well-known fluctuations in the conductance through a quantum dot in the Coulomb blockade regime [9, 10]: they result from the spatial structure of the quasi-bound wavefunction of the dot close to the leads and reflect how well this wavefunction couples to the electron states in the leads. We calculate the statistical distribution of the pumped current peak heights both in presence and absence of time-reversal symmetry (TRS). This leads to the prediction of an experimentally observable increase of the average pumped peak height upon breaking TRS.

Consider a quantum dot that is weakly-coupled to two single-mode leads via tunneling point contacts with transmission probabilities $T_{1}$ and $T_{2}$, see Fig. 1. If the strengths of the tunneling barriers are varied periodically as $V_{1}(t)=\bar{V}_{1}+\delta V_{1} \sin (\omega t)$ and $V_{2}(t)=\bar{V}_{2}+\delta V_{2} \sin (\omega t+$ $\phi)$, with $\delta V_{1,2} \ll \bar{V}_{1,2}$, a current is pumped into lead 1 which is given by, at zero temperature 画,

$$
\begin{aligned}
I_{1} & =\frac{\omega e}{2 \pi^{2}} \int_{A} d V_{1} d V_{2} \operatorname{Im}\left(\frac{\partial s_{11}^{*}}{\partial V_{1}} \frac{\partial s_{11}}{\partial V_{2}}+\frac{\partial s_{12}^{*}}{\partial V_{1}} \frac{\partial s_{12}}{\partial V_{2}}\right)(1 \mathrm{a}) \\
& \approx \frac{\omega e}{2 \pi} \sin \phi \delta V_{1} \delta V_{2} \operatorname{Im}\left(\frac{\partial s_{11}^{*}}{\partial V_{1}} \frac{\partial s_{11}}{\partial V_{2}}+\frac{\partial s_{12}^{*}}{\partial V_{1}} \frac{\partial s_{12}}{\partial V_{2}}() \mathrm{b}\right)
\end{aligned}
$$

(and $I_{2}=-I_{1}$ ). Eq. (11) is valid for slow perturbations such that $\omega \ll \tau_{\text {dwell }}^{-1}$, with $\tau_{\text {dwell }}$ the dwell time of particles in the dot, and $A$ denotes the area that is enclosed in parameter space $\left(V_{1}, V_{2}\right)$ during one period $\tau \equiv 2 \pi / \omega$. It relates the pumped current to the scattering matrix elements $s_{11}$ and $s_{12}$ of the dot, where $s_{\alpha \beta}$ denotes the scattering amplitude of electrons from lead $\beta$ to lead $\alpha$. The second equation (1b) is applicable for bilinear response to the perturbations $\delta V_{1}$ and $\delta V_{2}$, in which case the integral in (1a) becomes independent of the pumping contour [4]. This is the situation we consider here. In the low temperature regime $k_{B} T<\Gamma \ll \Delta$ transport occurs through a single bound state of the dot [8], which can then be modeled as a one-dimensional system with $\delta$-function barriers $V_{1} \delta(x)$ and $V_{2} \delta(x-L)$ [7, 11. The scattering matrix elements for this system are given by 


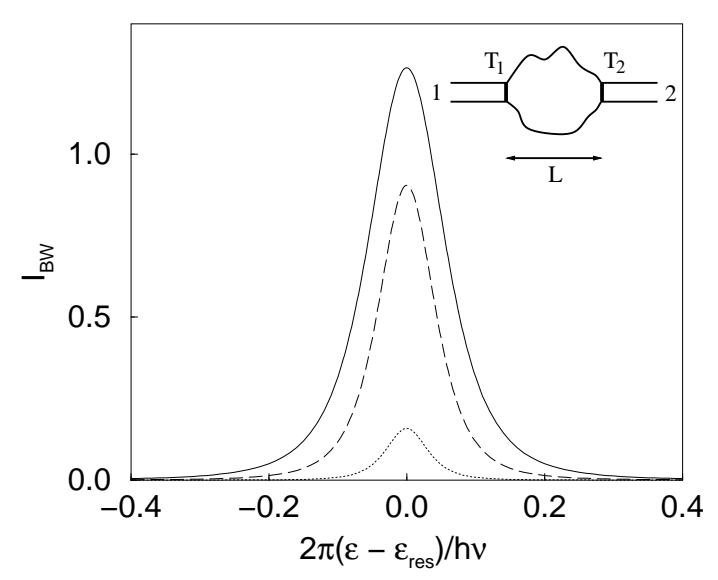

FIG. 1: Pumped current (9) in units of $\frac{\omega e}{4 \pi} \sin \phi \delta V_{1} \delta V_{2}$ through a nearly-closed quantum dot for $T_{1}=T_{2}=0.1$ (solid line), $T_{1}=0.1, T_{2}=0.05$ (dashed line), and $T_{1}=0.1, T_{2}=0.01$ (dotted line). Inset: Schematic picture of a quantum dot connected to two leads via tunneling barriers.

[7]

$$
\begin{aligned}
& s_{11}=r_{1}+\frac{r_{2} t_{1}^{2} e^{2 i k L}}{1-r_{1} r_{2} e^{2 i k L}} \\
& s_{12}=s_{21}=\frac{t_{1} t_{2} e^{i k L}}{1-r_{1} r_{2} e^{2 i k L}},
\end{aligned}
$$

with $k \equiv \sqrt{\frac{2 m}{\hbar}\left(\epsilon_{F}-\epsilon\right)}, \epsilon$ the energy measured from the Fermi energy $\epsilon_{F}$ and $s_{22}=s_{11}\left(r_{1} \leftrightarrow r_{2}, t_{1} \rightarrow t_{2}\right)$. Here $r_{m}$ and $t_{m}$ denote the reflection and transmission amplitudes of barrier $m[m=1,2]$,

$$
\begin{aligned}
r_{m} & =\frac{V_{m}}{i-V_{m}} \equiv \sqrt{R_{m}} e^{i \phi_{m}} \\
t_{m} & =\frac{i}{i-V_{m}} \equiv i \sqrt{T_{m}} e^{i \phi_{m}} \\
\phi_{m} & \equiv-\arctan V_{m}-\pi / 2,
\end{aligned}
$$

with $R_{m} \equiv\left|r_{m}\right|^{2}$, and $T_{m} \equiv\left|t_{m}\right|^{2}$. The integrand of Eq. (1a) can then be written as

$$
\begin{aligned}
\Pi\left(T_{1}, T_{2}\right) \equiv & \operatorname{Im}\left(\frac{\partial s_{11}^{*}}{\partial V_{1}} \frac{\partial s_{11}}{\partial V_{2}}+\frac{\partial s_{12}^{*}}{\partial V_{1}} \frac{\partial s_{12}}{\partial V_{2}}\right) \\
= & \frac{\left(T_{1} T_{2}\right)^{\frac{3}{2}}}{N}\left[( \sqrt { R _ { 1 } T _ { 2 } } + \sqrt { R _ { 2 } T _ { 1 } } ) \left(1-\sqrt{R_{1} R_{2}}\right.\right. \\
& \left.\cos \Theta)-\left(1-R_{1} R_{2}+\sqrt{R_{1} T_{1} R_{2} T_{2}}\right) \sin \Theta(]\right) .
\end{aligned}
$$

with

$$
\begin{aligned}
N & \equiv\left[\left(1-\sqrt{R_{1} R_{2}}\right)^{2}+2 \sqrt{R_{1} R_{2}}(1-\cos \Theta)\right]^{2} \\
\Theta & \equiv \phi_{1}+\phi_{2}+2 k L .
\end{aligned}
$$

For a nearly-closed quantum dot, the transmission probabilities $T_{1}, T_{2} \ll 1$. If $\delta V_{m} \ll V_{m}$ and hence $\delta T_{m}$ $\ll T_{m}$, for $m \in\{1,2\}$, the quantum dot remains in the Coulomb blockade regime during the entire pumping cycle. $\Pi$ then exhibits sharp resonances when $N=0$. For a completely-closed $\operatorname{dot}\left(T_{1}=T_{2}=0\right)$ the resonance condition is given by $\cos \Theta=1$. In case of a nearly-closed dot the cosine can be expanded around this resonance as $1-\cos \Theta \approx \frac{1}{2}\left(\frac{d \Theta}{d \epsilon}\right)^{2}\left(\epsilon-\epsilon_{\mathrm{res}}\right)^{2}$. Up to lowest order in $T_{1}$ and $T_{2}$ [12] the pumped current $I_{B W}$ is then given by

$$
I_{B W}=\frac{\omega e}{4 \pi} \sin \phi \delta V_{1} \delta V_{2} \frac{\left(T_{1} T_{2}\right)^{\frac{3}{2}}\left(T_{1}^{\frac{1}{2}}+T_{2}^{\frac{1}{2}}\right)\left(T_{1}+T_{2}\right)}{\left[\left(\frac{T_{1}+T_{2}}{2}\right)^{2}+\left(\frac{\epsilon-\epsilon_{\text {res }}}{\hbar \nu}\right)^{2}\right]^{2}},(9)
$$

with $\hbar \nu \equiv d \epsilon / d \Theta$ and $\nu$ the attempt frequency, the inverse of the round-trip travel time between the two barriers. Equation (99) is valid for $k_{B} T \ll \Gamma \ll \Delta,\left|\epsilon-\epsilon_{\text {res }}\right| \ll$ $\hbar \nu$, and bilinear response to the perturbations $\delta V_{1}$ and $\delta V_{2}$. Figure 11 shows that $I_{B W}$ is sharply peaked around the resonant level $\epsilon_{\text {res }}$ 13. The effect of finite temperatures can be incorporated by thermally averaging (9) as $I \equiv-\int d \epsilon I_{B W} f^{\prime}(\epsilon, T) \approx \frac{1}{4 k_{B} T} \int_{\epsilon_{\mathrm{res}}-\frac{1}{2} \hbar \nu\left(T_{1}+T_{2}\right)}^{\epsilon_{\mathrm{res}}} d \epsilon I_{B W}$, where $f(\epsilon, T) \equiv\left[1+\exp \left(\epsilon / k_{B} T\right)\right]^{-1}$ denotes the Fermi function. This results in the peak heights, for $\Gamma \ll$ $k_{B} T \ll \Delta$,

$$
I_{\max }=\frac{\omega e(2+\pi) \bar{\Gamma}^{3 / 2}}{16 \pi k_{B} T \sqrt{\hbar \nu}} \sin \phi \delta V_{1} \delta V_{2} \alpha .
$$

Here $\alpha$ denotes the amplitude of the current normalized by the mean resonance width $\bar{\Gamma}$,

$$
\alpha \equiv \frac{\left(\Gamma_{1} \Gamma_{2}\right)^{\frac{3}{2}}\left(\Gamma_{1}^{\frac{1}{2}}+\Gamma_{2}^{\frac{1}{2}}\right)}{\bar{\Gamma}^{\frac{3}{2}}\left(\Gamma_{1}+\Gamma_{2}\right)^{2}},
$$

with $\Gamma_{1,2} \equiv \hbar \nu T_{1,2}$. Equations (9) and (10) are the pumping analogs of, resp., the Breit-Wigner conductance $G_{\mathrm{BW}}$ through a quantum dot in the resonant tunneling regime [11] and its peak heights $G_{\max }$ [14],

$$
\begin{array}{rlrl}
G_{B W} & =\frac{2 e^{2}}{h} \frac{\Gamma_{1} \Gamma_{2}}{\frac{1}{4} \Gamma^{2}+\left(\epsilon-\epsilon_{\mathrm{res}}\right)^{2}}, & & T=0, \Gamma \ll \Delta(.12) \\
G_{\max }=\frac{e^{2}}{h} \frac{\pi}{4 k_{B} T} \frac{\Gamma_{1} \Gamma_{2}}{\Gamma_{1}+\Gamma_{2}}, & & \Gamma \ll k_{B} T \ll \Delta(13)
\end{array}
$$

Jalabert et al. [9] calculated the statistical distributions of the Coulomb blockade conductance peaks (13) for singlemode leads in presence and absence of time-reversal symmetry, which were in excellent agreement with subsequent experiments [10]. In an analogous manner we now proceed to calculate the distribution of the pumped current peaks (11). Assuming equivalent single-mode point contacts with energy-independent mean tunneling rates $\bar{\Gamma}_{1}=\bar{\Gamma}_{2} \equiv \bar{\Gamma} / 2$ (valid if the separation between the barriers $L \gg \lambda_{F}$, with $\lambda_{F}$ the Fermi wavelength), the distributions of $\Gamma_{1}$ and $\Gamma_{2}$ for a chaotic quantum dot are given by the Porter-Thomas distribution [9]

$$
P_{\beta}\left(\Gamma_{m}\right)=\left(\frac{\beta}{2 \bar{\Gamma}}\right)^{\beta / 2} \frac{1}{G(\beta / 2)} \Gamma_{m}^{\beta / 2-1} e^{-\beta \Gamma_{m} / 2 \bar{\Gamma}},
$$


[ $m=1,2]$, where $G$ denotes the Gamma function and the symmetry index $\beta=1(2)$ in the presence (absence) of time-reversal symmetry. For independently fluctuating tunneling rates the distribution of $\alpha$ is then given by $P_{\beta}(\alpha)=\int d \Gamma_{1} \int d \Gamma_{2} P_{\beta}\left(\Gamma_{1}\right) P_{\beta}\left(\Gamma_{2}\right) \delta(\alpha-$ $\left.\frac{\left(\Gamma_{1} \Gamma_{2}\right)^{\frac{3}{2}}\left(\sqrt{\Gamma_{1}}+\sqrt{\Gamma_{2}}\right)}{\bar{\Gamma}^{\frac{3}{2}}\left(\Gamma_{1}+\Gamma_{2}\right)^{2}}\right)$. In case of a symmetric dot with $\Gamma_{1}=\Gamma_{2}$ in zero magnetic field this yields

$$
P_{1, \Gamma_{1}=\Gamma_{2}}(\alpha)=\frac{4}{3 \sqrt{2 \pi}(2 \alpha)^{2 / 3}} e^{-\frac{1}{2}(2 \alpha)^{2 / 3}} .
$$

For the more general case of an asymmetric dot with $\Gamma_{1} \neq \Gamma_{2}$, it is convenient to write (11) as $\alpha \equiv \alpha_{1}+$ $\alpha_{2}=\frac{\Gamma_{1}^{2} \Gamma_{2}^{\frac{3}{2}}}{\bar{\Gamma}^{\frac{3}{2}}\left(\Gamma_{1}+\Gamma_{2}\right)^{2}}+\frac{\Gamma_{1}^{\frac{3}{2}} \Gamma_{2}^{2}}{\Gamma^{\frac{3}{2}}\left(\Gamma_{1}+\Gamma_{2}\right)^{2}}$, calculate the distributions $P_{\beta}\left(\alpha_{1}\right)$ and $P_{\beta}\left(\alpha_{2}\right)$ and from these $P_{\beta}(\alpha)=$ $\int d \alpha_{1} \int d \alpha_{2} P_{\beta}\left(\alpha_{1}\right) P_{\beta}\left(\alpha_{2}\right) \delta\left(\alpha-\left(\alpha_{1}+\alpha_{2}\right)\right)$. This results in the following expressions (exact for $P_{1}(\alpha)$ and numerical approximation to within $0.1 \%$ accuracy for $\left.P_{2}(\alpha)\right)$

$P_{1}(\alpha)=\frac{C_{1}}{\alpha^{\frac{1}{3}}} \int_{0}^{1} d x \frac{1}{[x(1-x)]^{\frac{2}{3}}} e^{-\frac{1}{2}\left(\frac{35}{3} \alpha\right)^{\frac{2}{3}}\left[x^{\frac{2}{3}}+(1-x)^{\frac{2}{3}}\right.}(16)$

$P_{2}(\alpha)=C_{2} \alpha^{\frac{4}{5}} \int_{0}^{1} d x \frac{1}{[x(1-x)]^{\frac{1}{10}}} e^{-\left(\frac{35}{3} \alpha\right)^{\frac{2}{3}}\left[x^{\frac{2}{3}}+(1-x)^{\frac{2}{3}}\right]}(17)$

with $C_{1} \equiv 2(35 / 3)^{\frac{2}{3}} / 9 \pi$ and $C_{2} \approx 46.6$. The distributions $P_{1}(\alpha)$ and $P_{2}(\alpha)$ are plotted in Fig. 2. Breaking TRS increases the mean amplitude from $\bar{\alpha}_{B=0}=\frac{12}{35} \sqrt{\frac{2}{\pi}}$ $\approx 0.27$ to $\bar{\alpha}_{B \neq 0}=\frac{4}{21} \sqrt{\pi} \approx 0.34$. Simultaneously, the variance of $\alpha$ decreases from $\sigma^{2}(\alpha)_{B=0}=\frac{15}{64}-\left(\frac{12}{35}\right)^{2} \frac{2}{\pi} \approx 0.16$ to $\sigma^{2}(\alpha)_{B \neq 0}=\frac{6}{35}-\left(\frac{4}{21}\right)^{2} \pi \approx 0.06$ [15]. The distributions (16) and (17) are similar to the ones for conductance in Ref. 91, with means and variances being of the same order of magnitude. Jalabert et al. tested their prediction of the Porter-Thomas distribution (14) numerically and obtained excellent agreement. This analysis also serves as a numerical test of the validity of $P_{1}(\alpha)$ and $P_{2}(\alpha)$, since these follow analytically from (14).

The above model is entirely based on a noninteracting particle picture. Since the charge distribution in a quantum dot is modified by interactions among the electrons |16], the question arises whether one may neglect the effect of e-e interactions on quantum pumping in the Coulomb blockade regime here. Measurements of the phase of the reflection coefficient of a quantum dot in the linear quantum Hall regime were observed to be in good agreement with a noninteracting model if the dot was close to resonance, with deviations occurring further away from the resonance condition [17]. Therefore the present noninteracting model is expected to give, at least in first approximation, a good description of quantum pumping (and the pumped peak heights) in a nearlyclosed dot close to resonance. Studying the effects of e-e

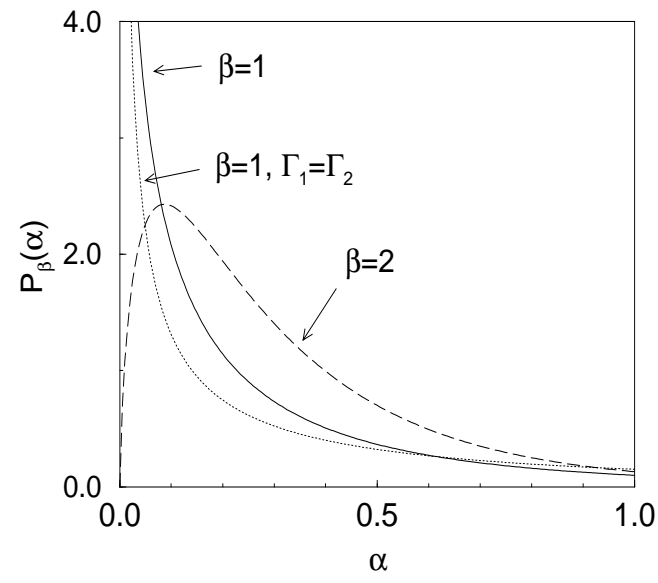

FIG. 2: Distribution $P_{\beta}(\alpha)$ of pumped current peak heights for symmetric leads in zero magnetic field $\left(P_{1, \Gamma_{1}=\Gamma_{2}}(\alpha)\right.$, dotted line), asymmetric leads in zero magnetic field $\left(P_{1}(\alpha)\right.$, solid line) and asymmetric leads in a magnetic field $\left(P_{2}(\alpha)\right.$, dashed line).

interactions in this regime forms an interesting direction for future research.

The change in $\bar{\alpha}$ upon application of a magnetic field can be experimentally observed in small strongly-pinched quantum dots with typical diameter $\sim 1 \mu \mathrm{m}$, Fermi wavelength $\lambda_{F} \sim 10 \mathrm{~nm}$, level spacing $\Delta \sim 10 \mu \mathrm{eV}$ and barrier conductances $G_{1,2} \ll \frac{2 e^{2}}{h}[18$. Adiabatic quantum pumping through the dot may be achieved at low temperatures $T \sim 10 \mathrm{mK}$, for which $\Gamma<k_{B} T<\Delta$, by periodically modulating either the tunneling barriers with two small-amplitude phase-shifted rf signals [2], or the confining potential of the dot by two shape-changing voltages. Measuring the pumped current peaks as a function of an additional split-gate voltage, which varies the electron density of the dot and hence the Fermi energy $\epsilon_{F}$, would then allow to assemble their statistics. The modulation amplitudes must be small enough for the perturbation of the energy levels of the dot to be $\ll \Delta$ for each value of $\epsilon_{F}$. Another important requirement is that the dephasing (loss of phase coherence) and leakage (escape of electrons through the leads) times must be larger than the resonant tunneling time. The former have been measured for nearly-closed quantum dots at temperatures $\sim 10 \mathrm{mK}$ in Ref. [18] and are given by $\tau_{\text {dephasing }} \sim 1 \mathrm{~ns}$ and $\tau_{\text {escape }} \sim 0.1 \mathrm{~ns}$, respectively. Since the typical dwell time for electrons on the $\operatorname{dot} \tau_{\text {dwell }} \sim 10^{-12}-10^{-11} \mathrm{~s}$ for $G_{1,2} \sim 0.01-0.1 e^{2} / h$, we expect that quantum pumping should not be strongly affected by these inelastic processes. For typical pumping frequencies $f \sim 10 \mathrm{MHz}$ [2. 6], also the adiabaticity condition $\omega=2 \pi f \ll \tau_{\text {dwell }}^{-1}$ is fullfilled.

Statistical theories based on RMT such as the above and in Ref. [9] do not account for correlations between adjacent peaks. In measurements of the conductance, 
however, these were observed [10, 19] and semiclassical analysis suggested that they result from short-time dynamics in the dot 20. An interesting direction for future research is therefore to study the correlations of pumped current peak heights. Another open question concerns the effects of dephasing on these peak heights. For conductance, the normalized change in average peak heights upon breaking TRS in the absence of dephasing $\delta g \equiv\left(\langle g\rangle_{B \neq 0}-\langle g\rangle_{B=0}\right) /\langle g\rangle_{B \neq 0}$ is independent of temperature [21]. $\delta g$ can thus be used to investigate the temperature dependence of the dephasing time, $\tau_{\phi}$, since any observed temperature dependence in measurements of $\delta g$ is due to $\tau_{\phi}[18$. Similarly the change in average pumped current peak heights may provide a useful tool to study dephasing.

In conclusion, we shave studied adiabatic quantum pumping and its statistics in the coherent resonant tunneling regime of a nearly-closed quantum dot. Using random-matrix theory, the average peak height of the pumped current is predicted to display an observable increase in the presence of a symmetry-breaking magnetic field.

Stimulating discussions with Y. Levinson and C.M. Marcus are gratefully acknowledged. This work was supported by the Netherlands Organisation for Scientific Research (NWO), by NFS grant CHE-0073544 and by an NSF MRSEC grant.

[1] D.J. Thouless, Phys. Rev. B 27, 6083 (1983).

[2] See e.g. the realizations of single-charge pumping in quantum dots by L.P. Kouwenhoven et al., Phys. Rev. Lett. 67, 1626 (1991); L.P. Kouwenhoven et al., Z. Phys. B 85, 381 (1991); H. Pothier et al., Europhys. Lett. 17, 249 (1992).

[3] The word "classical" here refers to the fact that these pumping mechanisms do not involve quantum interference, but are based on sequential tunneling processes in the Coulomb blockade regime of a quantum dot and thereby resemble classical peristaltic pumping.

[4] P.W. Brouwer, Phys. Rev. B 58, R10135 (1998).

[5] See e.g. B. Spivak et al., Phys. Rev. B 51, 13226 (1995); I.L. Aleiner and A.V. Andreev, Phys. Rev. Lett. 81, 1286 (1998); F. Zhou et al., Phys. Rev. Lett. 82, 608 (1999); T.A. Shutenko et al., Phys. Rev. B 61, 10366 (2000); I.L. Aleiner et al., Phys. Rev. B 62, 10373 (2000); J.E. Avron et al., Phys. Rev. B 62, 10618 (2000); A.V. Andreev and A. Kamenev, Phys. Rev. Lett. 85, 1294 (2000); M.G. Vavilov et al., Phys. Rev. B 63, 195313 (2001).

[6] M. Switkes et al., Science 283, 1905 (1999).

[7] Y. Levinson et al., condmat/0010494.

[8] See for a review on electron transport through quantum dots L.P. Kouwenhoven et al., in Proceedings of the Advanced Study Institute on Mesoscopic Electron Transport, L.P. Kouwenhoven, G. Schön, L.L. Sohn, Eds. (Kluwer, Dordrecht, 1997).

[9] R.A. Jalabert et al., Phys. Rev. Lett. 68, 3468 (1992); V.N. Prigodin et al., Phys. Rev. Lett. 71, 1230 (1993).

[10] A.M. Chang et al., Phys. Rev. Lett. 76, 1695 (1996); J.A. Folk et al., Phys. Rev. Lett. 76, 1699 (1996).

[11] A.D. Stone and P.A. Lee, Phys. Rev. Lett. 54, 1196 (1985).

[12] The complete expanded expression for $\Pi\left(T_{1}, T_{2}\right)$ is given by $\Pi\left(T_{1}, T_{2}\right)=\left(T_{1} T_{2}\right)^{3 / 2}\left[\frac{1}{2}\left(\sqrt{T_{1}}+\sqrt{T_{2}}\right)\left(T_{1}+T_{2}\right)-\right.$ $\left.\left(T_{1}+T_{2}+\sqrt{T_{1} T_{2}}\right)\left(\frac{\epsilon-\epsilon_{\mathrm{res}}}{\hbar \nu}\right)+\frac{1}{2}\left(\sqrt{T_{1}}+\sqrt{T_{2}}\right)\left(\frac{\epsilon-\epsilon_{\mathrm{res}}}{\hbar \nu}\right)^{2}\right]$ $/\left[\left(\frac{T_{1}+T_{2}}{2}\right)^{2}+\left(\frac{\epsilon-\epsilon_{\mathrm{res}}}{\hbar \nu}\right)^{2}\right]^{2}$.

[13] For lower barrier heights these resonances become more broadened, see Y. Wei et al., Phys. Rev. B 62, 9947 (2000).

[14] C.W.J. Beenakker, Phys. Rev. B 44, 1646 (1991).

[15] A similar decrease in variance has been observed in open quantum dots, see Ref. [6].

[16] See e.g. A.K. Evans et al., Phys. Rev. B 48, 11120 (1993).

[17] E. Buks et al., Phys. Rev. Lett. 77. 4664 (1996).

[18] See e.g. J.A. Folk et al., cond-mat/0008052.

[19] S.M. Cronenwett et al., Phys. Rev. Lett. 79, 2312 (1997); S.R. Patel et al., Phys. Rev. Lett. 81, 5900 (1998).

[20] E.E. Narimanov et al., Phys. Rev. Lett. 83, 2640 (1999); L. Kaplan, Phys. Rev. E 62, 3476 (2000).

[21] Y. Alhassid, Phys. Rev. B 58, R13383 (1998). 